\title{
CONTROLE FUZZY APLICADO À MELHORIA DA ESTABILIDADE DINÂMICA EM SISTEMAS ELÉTRICOS DE POTÊNCIA
}

\author{
Walter Barra \\ Junior \\ DEEC-CT-UFPA \\ Campus Universitário do \\ Guamá, CEP 66075-900, \\ Belém, PA, \\ Fone: (0xx91) 3183-1680, \\ e-mail: walbarra@ufpa.br
}

\author{
José Augusto Lima \\ Barreiros \\ DEEC-CT-UFPA \\ Campus Universitário do \\ Guamá, CEP 66075-900, \\ Belém, PA \\ Fone: (0xx91) 3183-1680, \\ e-mail: barreiro@ufpa.br
}

\section{Carlos Tavares da Costa Júnior \\ DEEC-CT-UFPA}

Campus Universitário do

Guamá, CEP 66075-900, Belém, PA

Fone: (0xx91) 3183-1680, e-mail:cartav@ufpa.br

\section{André Maurício \\ Damasceno Ferreira \\ CEFET-PA}

Av. Alm. Barroso 1155

Bairro do Marco, CEP: 66093-020, Belém, PA,Fone: (0xx91) 3211-0364 e-mail: andre@amazon.com.br

\begin{abstract}
This work presents a fuzzy control strategy which can be applied to improve the dynamic stability of electric power systems. The gains of fuzzy controller are smoothly adjusted by using interpolation among the gains of a set of previously designed local controllers, according to the current plant operating condition. In this way, the parameters of the fuzzy controller are automatically adjusted on-line, in order to cope with changes in the power system operating conditions. The performance of the fuzzy strategy was assessed by simulation studies, using a nonlinear machine infinite bus bar dynamic model. The results show that it is possible to improve the power system dynamic performance under time-variable operating conditions.in english. Please notice that the abstract in english comes first.
\end{abstract}

KEYWORDS: Fuzzy Control, Power System Stability.

\section{RESUMO}

Este trabalho propõe uma estratégia fuzzy, do tipo Rede de

Artigo Submetido em 04/08/03

1a. Revisão em 18/03/04;

2a. Revisão em 15/02/05;

Aceito sob recomendação do Editor Associado

Prof. Dr. Glauco Taranto
Controladores Locais, aplicável à melhoria da estabilidade dinâmica em sistemas elétricos de potência, visando compensar possíveis perdas de sintonia devido à ocorrência de variações nas condições operacionais da planta. A adaptação dos ganhos do controlador fuzzy é efetuada on-line, interpolando-se os ganhos de um conjunto finito de controladores locais fixos. Ao ocorrer variações nas condições operacionais da planta, os ganhos da lei de controle são ajustados automaticamente de modo a manter satisfatório o desempenho do sistema de controle. $\mathrm{O}$ desempenho do controle foi avaliado através de estudos de simulação, utilizando-se um modelo dinâmico não-linear, do tipo máquina barra infinita. Os resultados mostram que o emprego da estratégia proposta permite obter melhorias no desempenho dinâmico do sistema.

PALAVRAS-CHAVE: Controle Fuzzy, Dinâmica de Sistemas de Elétricos de Potência.

\section{INTRODUÇÃO}

A operação segura e eficiente de um sistema elétrico de potência depende do emprego de controladores automáticos em diferentes níveis hierárquicos, para garantir estabilidade de operação e a qualidade do produto. A maioria dos sistemas de controle atualmente um uso ainda são baseados em estruturas lineares com parâmetros fixos, denominados controladores convencionais. A razão 
para isso talvez ainda resida na maior familiaridade dos engenheiros com as técnicas de projeto e implementação de controladores convencionais.

Contudo, é um fato bem conhecido que as não-linearidades e condições operacionais variáveis, tipicamente presentes em sistemas elétricos de potência, podem provocar perda de sintonia em um sistema de controle com parâmetros fixos. Tais limitações motivaram um grande número de pesquisas na busca de novas estratégias de controle aptas a lidar com tal problema. Dentre as estratégias mais investigadas podemos citar aquelas baseadas em controle adaptativo auto-ajustável (Zachariah (1994), Farsi et al.(1997), Barreiros et al.(1998)), e aquelas baseadas em inteligência computacional, com destaque para as metodologias dos tipos fuzzy e neural (Flynn et al.(1997), Wang et al.(1995)).

Uma classe especial de sistemas fuzzy, denominada Rede de Modelos Locais (acrônimo LMN, em língua inglesa), a qual possui uma série de propriedades que facilitam a modelagem e o controle de sistemas não-lineares, tem atraído a atenção de um número crescente de pesquisadores. Em Zang e Morris (1995), os autores utilizaram LMN para efetuar a modelagem e o controle de processos químicos. Em Gollee e Hunt (1997), foi utilizada uma estratégia do tipo LMN para o estudo do comportamento dinâmico de um sistema muscular sob estímulo de impulsos elétricos. Em sistemas elétricos de potência, a estratégia LMN têm apresentado alguns resultados promissores(Prasad et al. (1998), Costa Jr. (1999), Barra Jr.( 2001)).

Este trabalho propõe uma estratégia de controle fuzzy, do tipo Rede de Controladores Locais (acrônimo LCN, em língua inglesa), destinada à melhoria do desempenho dinâmico de um sistema elétrico de potência, contribuindo para o amortecimento das oscilações do rotor. A estratégia implementa um tipo de "chaveamento" suave entre um conjunto de controladores locais previamente projetados, de acordo com a presente condição de operação da planta. Neste estudo, as condições operacionais da planta são determinadas das medidas das potências ativa e reativa nos terminais do gerador síncrono. $\mathrm{O}$ artigo está organizado da seguinte forma: na seção 2, apresenta-se uma breve introdução ao controle de sistemas dinâmicos utilizando-se LCN; na seção 3, apresenta-se a estratégia de controle proposta; na seção 4, são comentados alguns detalhes sobre o projeto do controlador fuzzy; na seção 5, são apresentados e discutidos os resultados obtidos em estudos de simulação; finalmente, na seção 6, apresentam-se as conclusões deste estudo.

\section{REDE DE CONTROLADORES LOCAIS}

Seja $S$ um sistema dinâmico não-linear, e suponha-se que exista um conjunto composto por $N_{O}$ variáveis mensuráveis, $o_{1}, \ldots, o_{N o}, \in R$, as quais caracterizam as condições operacionais de $S$ em um dado instante. Seja $O=\left[o_{1}, \ldots, o_{N o}\right]^{T}, \in R^{N o}$, definido como sendo um vetor contendo os valores instantâneos das variáveis de operação do sistema. Suponha-se, ainda, que o espaço de operação de $S$ possa ser particionado em $M$ regiões fuzzy ${ }^{1}$ onde, em cada uma dessas regiões, o comportamento dinâmico de $S$ possa ser aproximado localmente por um modelo linear, do tipo ARX, na forma

$$
\begin{aligned}
& A^{(l)}\left(q^{-1}\right) y(t)=q^{-d} B^{(l)}\left(q^{-1}\right) u(t)+c_{0}^{(l)}+e(t) \\
& l=1, \ldots, M
\end{aligned}
$$

onde $\mathrm{q}^{-1}$ repesenta o operador atraso de tempo discreto, definido como $\mathrm{q}^{-1} x(t)=x(t-1), y(t)$ é o sinal de saída de $S, u(t)$ é o sinal de entrada aplicado, e(t) é o erro de modelagem, $c_{0}^{(l)}$ é um termo de offset, $d$ é o atraso de transporte discreto, e $A^{(l)}\left(q^{-1}\right)$ e $B^{(l)}\left(q^{-1}\right)$ são os polinômios, na forma

$$
\begin{gathered}
A^{(l)}\left(q^{-1}\right)=1+a_{1}^{(l)} q^{-1}+a_{2}^{(l)} q^{-2}+\ldots+a_{N a}^{(l)} q^{-N a} \\
B^{(l)}\left(q^{-1}\right)=b_{0}^{(l)}+b_{1}^{(l)} q^{-1}+\ldots+b_{N b}^{(l)} q^{-N b}
\end{gathered}
$$

É suposto, ainda, que os coeficientes dos modelos locais possam ser obtidos através da linearização de um modelo não-linear de $S$ (se este for conhecido a priori), em torno de um dado ponto de operação, ou possam ser estimados através de testes de identificação, utilizando-se para isso um conjunto de medidas de entrada e saída da planta.

Para cada um dos modelos locais, pode-se projetar um correspondente controlador local, na forma RST (ver apêndice E)

$$
\begin{aligned}
& u^{(l)}(t)=\frac{1}{S^{(l)}\left(q^{-1}\right)}\left[T^{(l)}\left(q^{-1}\right) r(t)-R^{(l)}\left(q^{-1}\right) y(t)\right], \\
& l=1, \ldots, M
\end{aligned}
$$

onde $r(t)$ é o sinal de referência, e $R^{(l)}\left(q^{-1}\right), S^{(l)}\left(q^{-1}\right)$, e $T^{(l)}\left(q^{-1}\right)$, são polinômios em $q^{-1}$, expressos sob as formas

\footnotetext{
1 Neste artigo, o termo "região fuzzy" será utilizado para descrever regiões de operação cujas fronteiras são definidas através de funções de pertinência que indicam uma transição suave entre regiões vizinhas.
} 


$$
\begin{aligned}
& R^{(l)}\left(q^{-1}\right)=r_{0}^{(l)}+r_{1}^{(l)} q^{-1}+\ldots+r_{N r}^{(l)} q^{-N r} \\
& S^{(l)}\left(q^{-1}\right)=1+s_{1}^{(l)} q^{-1}+\ldots+s_{N s}^{(l)} q^{-N s} \\
& T^{(l)}\left(q^{-1}\right)=t_{0}^{(l)}=\frac{A^{(l)}(1)}{B^{(l)}(1)}\left[S^{(l)}(1)+R^{(l)}(1)\right]
\end{aligned}
$$

onde $T^{(l)}\left(q^{-1}\right)$ é selecionado de modo a garantir um ganho unitário em regime, para o sistema em malha fechada.

Neste trabalho, os coeficientes dos controladores locais podem ser calculados utilizando-se a técnica de projeto por posicionamento de pólos (Ästrom e Witennmark (1997)). Neste caso, os valores dos coeficientes dos controladores locais são obtidos da solução do seguinte sistema linear de equações

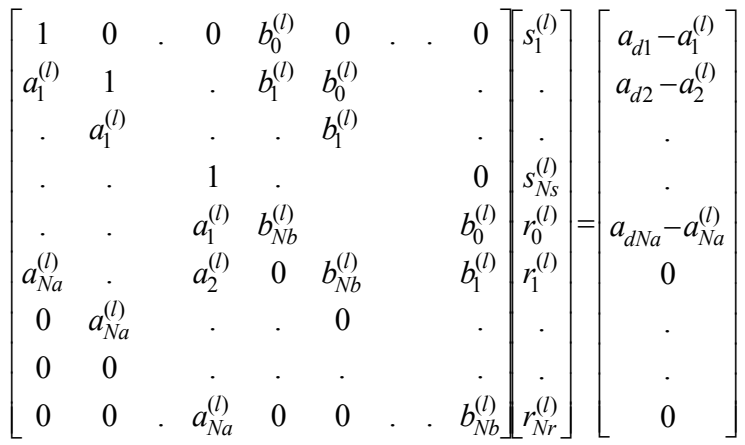

onde $a_{i}^{(l)}$ e $b_{j}^{(l)}, \mathrm{i}=1,2, . ., \mathrm{Na}, \mathrm{j}=0,1, \ldots, \mathrm{Nb}, \mathrm{e} \mathrm{l}=1,2, . ., \mathrm{M}$, são os coeficientes dos modelos locais da planta, e $a_{d i}$, $\mathrm{i}=1,2, \ldots, \mathrm{Na}$, são os coeficientes do polinômio desejado. $\mathrm{O}$ polinômio desejado deverá ser especificado pelo projetista, de acordo com o comportamento desejado para o sistema em malha fechada.

Uma vez calculados os parâmetros dos controladores locais, pode-se então definir-se uma lei de controle fuzzy, através do seguinte conjunto de regras fuzzy

$$
\begin{aligned}
& R^{(l)}: I F O(t) \text { is } O^{(l)} \\
& \text { THEN } u^{(l)}(t)=-S^{*(l)}\left(q^{-1}\right) u(t)+T^{(l)}\left(q^{-1}\right) r(t)-R^{(l)}\left(q^{-1}\right) y(t) \\
& l=1,2, \ldots, M
\end{aligned}
$$

onde $O(t)$ é o vetor cujas componentes são as variáveis de operação da planta, e $S^{*(l)}\left(q^{-1}\right)$ é um polinômio definido como $S^{*(l)}\left(q^{-1}\right)=S^{(l)}\left(q^{-1}\right)-1$.

Com base na teoria de sistemas fuzzy (Wang (1995)), a saída do sistema fuzzy definido pela equação (5), que no caso é o próprio sinal de controle, pode ser expressa sob a forma de uma média ponderada das saídas individuais dos controladores locais, ou seja

$$
u(t)=\frac{\sum_{l=1}^{M} \rho^{(l)}(O(t)) u^{(l)}(t)}{\sum_{l=1}^{M} \rho^{(l)}(O(t))}
$$

onde os pesos $\rho^{(l)}(O(t))$ são denominados de funções de validação. Estes pesos determinam os respectivos graus com que cada controlador local contribuirá para a saída do controlador fuzzy, em um dado instante, sendo que seus valores dependem da presente condição de operação da planta.

Os valores das funções de validação, em (6), são calculados on-line, a partir das medidas das variáveis de operação da planta e das funções de pertinência $\mu_{o_{1}}^{(l)}\left(o_{1}\right), \mu_{o_{2}}^{(l)}\left(o_{2}\right) \ldots \mu_{o_{N o}}^{(l)}\left(o_{N o}\right), \quad l=1, \ldots, M$, selecionadas pelo projetista. Isto é efetuado utilizando-se uma inferência do tipo produto, na forma

$$
\rho^{(l)}(O(t))=\mu_{o_{1}}^{(l)}\left(o_{1}(t)\right) \mu_{o_{2}}^{(l)}\left(o_{2}(t)\right) \ldots \mu_{o_{N o}}^{(l)}\left(o_{N o}(t)\right)
$$

É possível mostrar-se que a lei de controle fuzzy (equação 5) pode ser representada compactamente sob a seguinte forma polinomial:

$$
u(t)=-S^{*}\left(q^{-1}, O(t) \mu(t)+T\left(q^{-1}, O(t)\right) r(t)-R\left(q^{-1}, O(t)\right) y(t)\right.
$$

onde os polinômios $S^{*}\left(q^{-1}, O\right), R\left(q^{-1}, O\right)$ e $T\left(q^{-1}, O\right)$, têm formas semelhantes àquelas fornecidas nas equações (3.1), (3.2) e (3.3), respectivamente.

Os coeficientes do controlador fuzzy podem ser calculados, a cada intervalo de amostragem, utilizando-se as seguintes fórmulas de defuzzyficação pela média dos centros:

$$
\begin{array}{r}
s_{i}^{*}(O(t))=\frac{\sum_{l=1}^{M} \rho^{(l)}(O(t)) s_{i}{ }^{(l)}}{\sum_{l=1}^{M} \rho^{(l)}(O(t))} ; i=1,2, \ldots, N_{s} \\
r_{j}(O(t))=\frac{\sum_{l=1}^{M} \rho^{(l)}(O(t)) r_{j}{ }^{(l)}}{\sum_{l=1}^{M} \rho^{(l)}(O(t))} \quad ; j=0,1, \ldots, N_{r} \\
t_{0}(O(t))=\frac{A(1, O(t))}{B(1, O(t))}[S(1, O(t))+R(1, O(t))]
\end{array}
$$

É importante observar que os coeficientes do controlador fuzzy dependem tanto dos coeficientes dos controladores locais quanto dos valores assumidos pelas funções de 
validação no presente ponto de operação da planta. Em resumo, os ganhos do controlador fuzzy irão ajustar-se de acordo com as mudanças detectadas nas condições operacionais da planta.

Os ganhos dos controladores locais são, neste caso, calculados previamente durante a fase de projeto (utilizando-se para isso a equação (4)). Estes valores são subsequentemente armazenados em uma tabela para sua posterior utilização, durante a operação on-line do controlador fuzzy. Os valores das funções de validação, por sua vez, deverão ser calculados on-line, das medidas instantâneas das variáveis de operação. Dessa forma, para

a estratégia proposta neste trabalho, as únicas grandezas que necessitariam ser adquiridas on-line são as variáveis de operação e o sinal de saída a ser controlado.

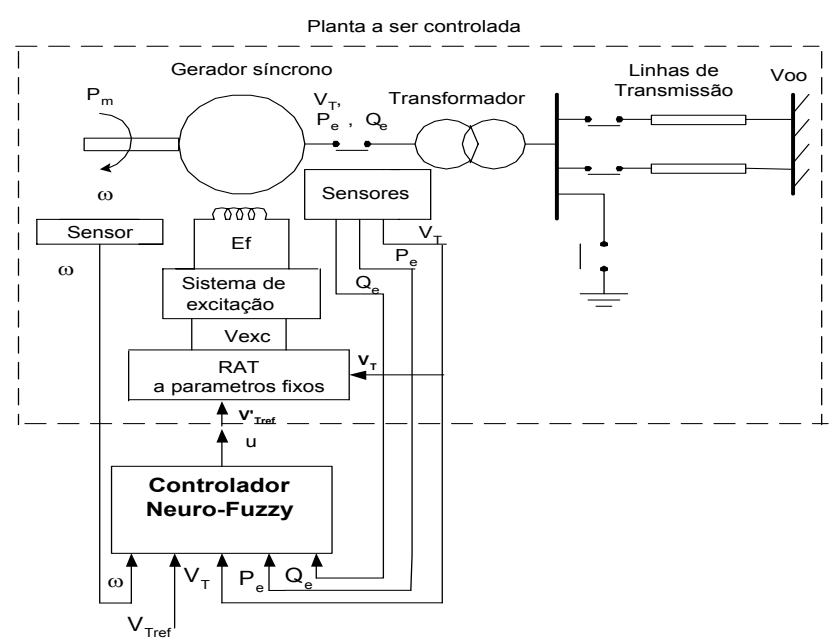

Figura 1. Sistema de Potência e controlador fuzzy.

\section{DESCRIÇÃO DO SISTEMA DE POTÊNCIA E OBJETIVOS DO CONTROLE PROPOSTO}

A planta simulada (sistema elétrico de potência), utilizada nos testes, consiste de um gerador síncrono interligado a um sistema de grande porte (denominado de barramento infinito), através de uma linha de transmissão dupla (figura 1). A planta a ser controlada, neste caso, é composta pelo gerador síncrono interligado, incluindo-se ainda o regulador automático de tensão (RAT). Os objetivos do controle fuzzy são:

melhorar o desempenho do regulador automático de tensão, de modo a obter-se um desempenho aproximadamente uniforme em toda a faixa de operação da planta; (ii)

melhorar o amortecimento das oscilações dinâmicas do rotor, de modo a obter-se um aumento nas margens de estabilidade dinâmica da planta.

A atuação do controlador fuzzy é feita através da modulação do valor do sinal de referência do RAT fixo. Os sinais de entrada para o controlador fuzzy são: a tensão nos terminais do gerador, $V_{T}$, e a velocidade angular do rotor $\omega$. As medidas das potências ativa e reativa nos terminais do gerador, $P_{e} \mathrm{e} Q_{e}$, serão utilizadas para detectar a condição operacional da planta.

Para simular-se a dinâmica não-linear do gerador síncrono foi utilizado um modelo não-linear de $5^{\text {a }}$ ordem, sob a forma de equações de estados (Arrilaga e Watson (2001)). Este modelo não-linear é caracterizado por um conjunto de parâmetros físicos cujos valores serão supostos como sendo não conhecidos para fins de projeto do controlador fuzzy.

A modelagem do sistema de excitação do gerador foi feita através de uma função de transferência de $1^{\text {a }}$ ordem, com limitadores na saída. O RAT fixo foi simulado através do uso de um controlador PID digital, o qual foi projetado para fornecer, na condição de operação em vazio, e com tensão terminal e freqüência em seus valores nominais, um amortecimento relativo de $\xi=0.7$ e uma freqüência natural de $4 \pi \mathrm{rad} / \mathrm{seg}$. O intervalo de amostragem utilizado para o RAT fixo foi $T_{S}=0.020$ segundos.

Os dados utilizados nos estudos de simulação estão localizados nos apêndices A e B. O modelo dinâmico do gerador pode ser encontrado no apêndice A, enquanto que os valores dos parâmetros de gerador, linha de transmissão e do RAT, utilizados nos estudos de simulação deste artigo, estão localizados no apêndice B.

O projeto do controlador fuzzy foi baseado no uso de modelos locais, obtidos através de técnicas de estimação paramétrica a partir de medidas de entrada e de saída da planta. A principal vantagem desse enfoque é poder aliviar o projetista da necessidade de ter um conhecimento prévio muito detalhado sobre os valores dos parâmetros físicos e da configuração do sistema de potência, o que nem sempre é possível em situações reais. Isto é muito importante, do ponto de vista prático, uma vez que nem sempre os valores dos parâmetros físicos do sistema são conhecidos com um suficiente grau de confiabilidade para fins de utilização em projeto de controladores. 


\section{PROJETO DO CONTROLADOR FUZZY PARA SISTEMAS DE POTÊNCIA}

Nesta seção, são discutidos os critérios guias adotados na seleção das variáveis de projeto do controlador fuzzy, incluindo-se a seleção do grau de refinamento da partição do espaço de operação, o número de controladores locais necessários, e a seleção das funções de pertinência ( forma e grau de superposição). Em muitas aplicações de controle fuzzy, a seleção dessas variáveis de projeto tem sido feita de forma qualitativa pelos projetistas. Entretanto, este é um tópico que necessita ser estudado com mais profundidade. Deve-se buscar o estabelecimento de metodologias que permitam uma seleção mais sistemática dessas variáveis de projeto, objetivando-se não comprometer a estabilidade do sistema em malha fechada e, ainda, preservar a capacidade de generalização do controlador fuzzy.

\subsection{Impacto da Seleção das Variáveis de Projeto na Estabilidade do Sistema}

As variáveis de projeto, do controlador fuzzy, são, basicamente, o grau de refinamento utilizado na partição do espaço de operação (ou seja, a quantidade e localizações dos controladores locais no espaço de operação da planta), e a seleção dos correspondentes conjuntos fuzzy e suas respectivas funções de pertinência (definidas no espaço das variáveis de operação). Tais variáveis de projeto têm sido normalmente selecionadas pelos projetistas com base apenas em um conhecimento qualitativo sobre o comportamento da planta. Entretanto, foi demonstrado recentemente (Hunt e Johansen (1997)), que a seleção dessas variáveis de projeto tem um forte impacto na estabilidade do sistema em malha fechada.

Os efeitos colaterais de um particionamento demasiadamente grosseiro do espaço de operação (ou seja, o uso de um número demasiadamente pequeno de controladores locais), bem como a seleção inadequada de funções de pertinência, com um elevado grau de superposição entre regiões fuzzy não adjacentes, podem dar origem ao surgimento de uma dinâmica não modelada que, quando realimentada, poderia até mesmo desestabilizar o sistema em malha fechada.

\subsection{Considerações Práticas Sobre a Seleção das Variáveis de Projeto do Controlador Fuzzy}

Se por um lado não é recomendável efetuar-se um particionamento demasiadamente grosseiro do espaço de operação (pelas razões expostas acima, na seção (4.1)), por outro lado, não é muito efíciente, do ponto de vista computacional, efetuar-se o oposto, ou seja, um particionamento demasiadamente refinado do espaço de operação, já que isto implicaria em um aumento desnecessário na complexidade do controlador fuzzy. Como uma conseqüência desse aumento de complexidade, haveria um aumento considerável tanto na quantidade de memória utilizada quanto no tempo necessário à realização dos cálculos on-line.

De um ponto de vista prático, uma das principais justificativas para se utilizar um controlador inteligente, do tipo fuzzy, reside na possibilidade de se fazer uso de sua capacidade de generalização (ou seja, da capacidade do controlador em gerar um número de respostas que exceda ao repertório de respostas produzidas individualmente pelos controladores locais armazenados na base). Se o projetista selecionar funções de pertinência que apresentem um grau de superposição demasiadamente reduzido, esta capacidade de generalização do controlador poderá ser drasticamente diminuida, o que, como conseqüência, implicaria na necessidade de se ter um número maior de controladores locais na base.

\subsection{Escolha Adotada para as Variáveis de Projeto do Controlador Fuzzy}

As variáveis de operação selecionadas neste trabalho foram as potências ativa e reativa nos terminais do gerador, $P_{e}$ e $Q_{e}$. Tal seleção é justificada pelo fato de que $P_{e} \quad$ e $Q_{e}$ são normalmente suficientes para caracterizar uma determinada condição operacional da planta estudada. Uma das razões para isso é que, durante operação em regime permanente, os valores médios das variáveis de saída $\mathrm{V}_{\mathrm{T}}$ e $\omega$, desviam-se muito pouco de seus valores nominais, enquanto $P_{e}$ e $Q_{e}$ assumem valores bastantes distintos nas diversas condições de operação do sistema de potência. Dessa forma, $V_{T}$ e $\omega$ podem ser, a princípio, desconsideradas para fins de sua utilização como variáveis de operação.

Levando-se em consideração os compromissos existentes entre estabilidade em malha fechada, velocidade de execução on-line e a manutenção da capacidade de generalização do controlador, optou-se então pela seleção de funções de pertinência tendo suporte local, e sob as formas triangular e trapezoidal. $\mathrm{O}$ grau de superposição adotado foi de 50 porcento para regiões imediatamente adjacentes, no espaço de operação, e de zero porcento para as regiões não adjacentes.

Esta escolha conduz não apenas a uma razoável manutenção da capacidade de generalização do controlador, como também contribui para minimizar o efeito da dinâmica não modelada. Uma outra vantagem 
dessa escolha é que a quantidade de cálculos on-line necessários torna-se bastante reduzida, implicando em uma execução mais veloz da lei de controle fuzzy. A cada instante de amostragem, apenas $2^{\mathrm{N}}$ funções de validação necessitarão ser calculadas, onde $N$ é número de variáveis de operação utilizadas. Por exemplo, para duas variáveis de operação $(N=2)$, apenas quatro valores de função de ativação terão que ser calculados a cada intervalo de amostragem.

Através de uma análise qualitativa dos padrões de comportamento da tensão terminal, em uma série de testes de resposta ao degrau na tensão de referência, foi observado que o efeito das oscilações do rotor mostraramse muito mais intensos nas regiões do plano $\mathrm{PxQ}$ caracterizadas qualitativamente como tendo " $P_{e}=$ alto" $\mathrm{e}$ " $Q_{e}=$ negativo ou quase zero". Dessa forma, efetuou-se um particionamento fuzzy mais refinado nessas regiões.

O particionamento adotado está representado nas figuras 2 e 3 , onde pode ser observado que optou-se pela escolha de seis conjuntos fuzzy para caracterizar qualitativamente a variável de operação $P_{e}$, e de dez conjuntos fuzzy para caracterizar qualitativamente a variável de operação $Q_{e}$. Dessa forma, o espaço de operação foi repartido em um total 60 regiões de operação (tabela 1), tendo sido projetados correspondentes controladores locais para cada uma dessas regiões de operação.

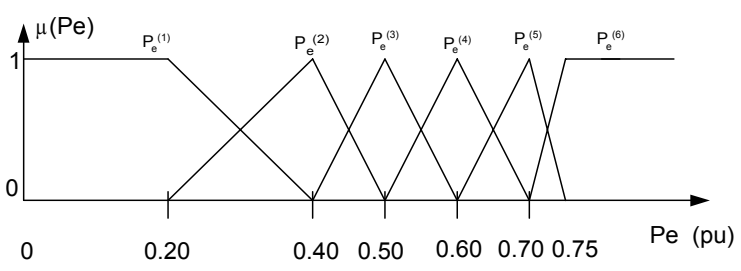

Figura 2. Particionamento fuzzy adotado para a variável de operação $P_{e}$.

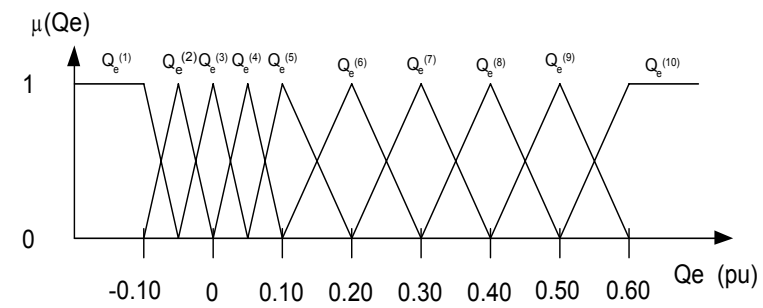

Figura 3. Particionamento fuzzy adotado para a variável de operação $Q_{e}$.
Tabela 1. Pontos de Operação (em p.u.)

\begin{tabular}{|c|c|c|c|c|c|c|}
\hline & $P_{e}^{(1)}$ & $\mathrm{P}_{\mathrm{e}}^{(2)}$ & $P_{e}^{(3)}$ & $P_{e}^{(4)}$ & $P_{e}^{(5)}$ & $P_{e}^{(6)}$ \\
\hline $\mathrm{Q}_{\mathrm{e}}^{(1)}$ & $\begin{array}{l}(0.20 \\
-0.10)\end{array}$ & $\begin{array}{l}(0.40, \\
-0.10)\end{array}$ & $\begin{array}{l}(0.50, \\
-0.10)\end{array}$ & $\begin{array}{l}(0.60, \\
-0.10)\end{array}$ & $\begin{array}{l}(0.20, \\
-0.10)\end{array}$ & $\begin{array}{l}(0.20, \\
-0.10)\end{array}$ \\
\hline $\mathrm{Q}_{\mathrm{e}}^{(2)}$ & $\begin{array}{l}(0.20 \\
-0.05)\end{array}$ & $\begin{array}{l}(0.40, \\
-0.05)\end{array}$ & $\begin{array}{l}(0.50 \\
-0.05)\end{array}$ & $\begin{array}{c}(0.60, \\
-0.05)\end{array}$ & $\begin{array}{l}(0.20 \\
-0.05)\end{array}$ & $\begin{array}{c}(0.20, \\
-0.05)\end{array}$ \\
\hline $\mathrm{Q}_{\mathrm{e}}^{(3)}$ & $\begin{array}{l}(0.20 \\
0.00)\end{array}$ & $\begin{array}{l}(0.40 \\
0.00)\end{array}$ & $\begin{array}{l}(0.50 \\
0.00)\end{array}$ & $\begin{array}{l}(0.60, \\
0.00)\end{array}$ & $\begin{array}{l}(0.20 \\
0.00)\end{array}$ & $\begin{array}{l}(0.20, \\
0.00)\end{array}$ \\
\hline $\mathrm{Q}_{\mathrm{e}}^{(4)}$ & $\begin{array}{l}(0.20 \\
0.05)\end{array}$ & $\begin{array}{l}(0.40 \\
0.05)\end{array}$ & $\begin{array}{l}(0.50 \\
0.05)\end{array}$ & $\begin{array}{l}(0.60 \\
0.05)\end{array}$ & $\begin{array}{l}(0.20 \\
0.05)\end{array}$ & $\begin{array}{l}(0.20, \\
0.05)\end{array}$ \\
\hline $\mathrm{Q}_{\mathrm{e}}^{(5)}$ & $\begin{array}{l}(0.20 \\
0.10)\end{array}$ & $\begin{array}{l}(0.40 \\
0.10)\end{array}$ & $\begin{array}{l}(0.50 \\
0.10)\end{array}$ & $\begin{array}{l}(0.60 \\
0.10)\end{array}$ & $\begin{array}{l}(0.20 \\
0.10)\end{array}$ & $\begin{array}{l}(0.20, \\
0.10)\end{array}$ \\
\hline $\mathrm{Q}^{(6)}$ & $\begin{array}{l}(0.20 \\
0.20)\end{array}$ & $\begin{array}{l}(0.40 \\
0.20)\end{array}$ & $\begin{array}{l}(0.50 \\
0.20)\end{array}$ & $\begin{array}{l}(0.6, \\
0.20)\end{array}$ & $\begin{array}{l}(0.20 \\
0.20)\end{array}$ & $\begin{array}{l}(0.20, \\
0.20)\end{array}$ \\
\hline $\mathrm{Q}_{\mathrm{e}}^{(7)}$ & $\begin{array}{l}(0.20 \\
0.30)\end{array}$ & $\begin{array}{l}(0.40 \\
0.30)\end{array}$ & $\begin{array}{l}(0.50 \\
0.30)\end{array}$ & $\begin{array}{l}(0.60, \\
0.30)\end{array}$ & $\begin{array}{l}(0.20 \\
0.30)\end{array}$ & $\begin{array}{l}(0.20, \\
0.30)\end{array}$ \\
\hline $\mathrm{Q}_{\mathrm{e}}^{(8)}$ & $\begin{array}{l}(0.20 \\
0.40)\end{array}$ & $\begin{array}{l}(0.40 \\
0.40)\end{array}$ & $\begin{array}{l}(0.50 \\
0.40)\end{array}$ & $\begin{array}{l}(0.20 \\
0.40)\end{array}$ & $\begin{array}{l}(0.20 \\
0.40)\end{array}$ & $\begin{array}{l}(0.20, \\
0.40)\end{array}$ \\
\hline $\mathrm{Q}_{\mathrm{e}}{ }^{(9)}$ & $\begin{array}{l}(0.20 \\
0.50)\end{array}$ & $\begin{array}{l}(0.40 \\
0.50)\end{array}$ & $\begin{array}{l}(0.50 \\
0.50)\end{array}$ & $\begin{array}{l}(0.20 \\
0.50)\end{array}$ & $\begin{array}{l}(0.20 \\
0.50)\end{array}$ & $\begin{array}{l}(0.20 \\
0.50)\end{array}$ \\
\hline $\mathrm{Q}^{(10)}$ & $\begin{array}{l}(0.20 \\
0.60)\end{array}$ & $\begin{array}{l}(0.40 \\
0.60)\end{array}$ & $\begin{array}{l}(0.50 \\
0.60)\end{array}$ & $\begin{array}{l}(0.20 \\
0.60)\end{array}$ & $\begin{array}{l}(0.20 \\
0.6)\end{array}$ & $\begin{array}{l}(0.20, \\
0.60)\end{array}$ \\
\hline
\end{tabular}

\subsection{Projeto dos Controladores Locais}

Em sistemas elétricos de potência operando de forma interligada, as oscilações dinâmicas de interesse estão normalmente localizadas na faixa $0.2<f<2$ hertz, aproximadamente. Assim, para excitar-se os modos de oscilação nesta faixa de freqüências, de modo a identificarse corretamente os coeficientes dos modelos locais da planta, utilizou-se um sinal de teste do tipo seqüência binária pseudo-aleatória (acrônimo PRBS, em língua inglesa), de $7^{\mathrm{a}}$ ordem, com um período de deslocamento de shift-register de 0.100 segundos. Com tal seleção, para o sinal de teste, a faixa de freqüências excitada de modo uniforme corresponde a $0.079<f<4.4$ hertz, aproximadamente. Observe que a faixa das oscilações dinâmicas $(0.2$ a $2 \mathrm{~Hz})$ está contida na faixa excitada de maneira uniforme pelo sinal PRBS projetado.

O sinal de teste PRBS foi aplicado de forma superposta ao valor de referência do RAT fixo. Este teste foi repetido para cada uma das 60 condições de operação selecionadas. Em cada teste, foram registrados, em um intervalo de tempo de 100 segundos, os dados de entrada e de saída da planta, que, neste caso, foram os desvios na tensão 
terminal e na velocidade angular do rotor. Os dados adquiridos foram, subsequentemente, utilizados na estimação dos parâmetros dos os modelos locais. Para isso, utilizou-se o conhecido método dos mínimos quadrados não-recursivo.

Para cada condição de operação selecionada, foram identificados dois modelos locais: um modelo relacionando o sinal de teste com o desvio na tensão terminal e um outro relacionando o sinal de teste com o desvio na velocidade angular. Utilizando-se modelos com estruturas lineares ARX, $\operatorname{com} d=1$ (atraso discreto igual a um intervalo de amostragem), o com intervalo de amostragem $T_{S}=0.020$ segundos, foram identificados modelos de $2^{\mathrm{a}}, 3^{\mathrm{a}}, 4^{\mathrm{a}}$ e $5^{\mathrm{a}}$ ordens para a planta.

Foi observado que os modelos de segunda ordem mostraram-se insatisfatórios, para fins de controle, porque apresentaram uma valor seperestimado para o amortecimento do modo de oscilação dominante. Já os modelos de ordem mais elevada, como os de $4^{\mathrm{a}}$ e $5^{\mathrm{a}}$ ordens, por sua vez, permitiram obter uma estimativa bastante precisa da dinâmica do sistema, apresentando um excelente desempenho em testes de validação baseados na análise de correlação do resíduo.

No entanto, analisando-se os padrões de pólos e zeros para os modelos acima da $4^{\mathrm{a}}$ ordem, observou-se a existência de, pelo menos, um par de pólos e de zeros muito próximos, quase cancelando-se mutuamente. Em Aström e Wittenmark (1997), é mostrado que o uso de modelos com pólos e zeros muito próximos pode resultar em problemas de condicionamento numérico na solução do sistema de equações (4), implicando em erros grosseiros no cálculo dos parâmetros do controlador.

Os modelos de $3^{\mathrm{a}}$ ordem, por sua vez, foram os que mostraram a melhor solução de compromisso pois, além de apresentarem um bom desempenho nos testes de validação, estes não apresentaram o problema de ter pólos e zeros muito próximos, resultando, portanto, em matrizes numericamente bem condicionadas para o cálculo dos parâmetros do controlador. Dessa forma, optou-se por adotar modelos de $3^{\mathrm{a}}$ para representar a dinâmica local do sistema, nas condições de operação investigadas.

Utilizando-se a técnica de projeto por posicionamento de pólos, foram então projetados os controladores locais, de modo a fornecer um desempenho uniforme, da regulação da tensão terminal, para cada condição de operação selecionada. Os requisitos de desempenho especificados para os controladores locais, foram os seguintes: amortecimento relativo desejado $\xi=0.7$, e freqüência angular natural $\omega_{n}=4 \pi \mathrm{rad} / \mathrm{s}$, para todas as condições de operação selecionadas.

\subsection{Adição de Uma Ação Estabilizadora Para Amortecer as Oscilações Dinâmicas}

Uma forma de se implementar o amortecimento das oscilações do rotor consiste em adicionar-se uma ação estabilizadora ao próprio regulador automático de tensão. Para isso, o valor da variável de realimentação do RAT (o valor da tensão terminal) é modificado pela adição de um termo proporcional ao desvio de velocidade $\Delta \omega$, na forma (Farsi et al., (1997)).

$$
y(t)=V_{T}(t)+\gamma \Delta \omega(t), \quad-1<\gamma<0
$$

onde $\gamma$ é um escalar utilizado para introduzir um certo grau de realimentação da variável estabilizante $\Delta \omega$.

A lógica por trás da criação dessa saída modificada é tentar sintetizar um sinal de saída $\mathrm{y}(\mathrm{t})$ que possua uma maior observabilidade em relação ao modo de oscilação dominante do que aquela normalmente presente no sinal original, $\mathrm{V}_{\mathrm{T}}$. Isto é feito através de uma combinação linear do sinal original com um sinal que possua um bom grau de observabilidade em relação ao modo de oscilação eletromecânica, como é o caso do desvio na velocidade angular do rotor, $\Delta \omega$. Nos testes apresentados, a saída modificada foi aplicada somente ao controlador fuzzy, enquanto que para o regulador de tensão fixo utilizou-se o sinal não modificado da tensão terminal $\mathrm{V}_{\mathrm{T}}$.

Devido ao atraso introduzido pelo sistema de excitação e gerador, a parcela de torque elétrico, resultante da realimentação estática de $\Delta \omega$, conforme a equação (9), não irá produzir um componente de torque de amortecimento puro. No entanto, é possível decompor a parcela de torque resultante em duas componentes: uma componente que estará em fase com $\Delta \omega$ e, portanto, contribuirá para aumentar o amortecimento da oscilação eletromecânica do rotor, e uma outra componente que estará em fase com os desvios do ângulo de carga, $\Delta \delta$, e que, portanto, afetará o torque de sincronismo.

Para atrasos de fase inferiores a 30 graus, aproximadamente, a componente em fase com $\Delta \omega$ será predominante, contribuindo assim para a melhoraria do amortecimento das oscilações eletromecânicas, ao preço de uma alteração no torque de sincronismo. Já para os atrasos de fase superiores a 30 graus, não seria recomendável utilizar-se uma realimentação estática de $\Delta \omega$, da forma apresentada na equação (9), porque a contribuição para o torque de amortecimento seria extremamente pobre e o torque sincronizante do sistema poderia ser adversamente afetado.

Neste caso, a medida do sinal $\Delta \omega$ deveria ser primeiramente processada por um filtro para compensar o 
atraso de fase, produzindo-se assim um sinal modificado o qual poderia então ser utilizado no lugar de $\Delta \omega$, na equação (9).

Em Farsi et al. (1997), a escolha do valor utilizado para o fator $\gamma$ parece ter sido feita por meio de tentativa e erro. Os autores ressaltaram que, devido ao caráter não-linear da planta, não seria uma tarefa simples a escolha de um único valor do coeficiente $\gamma$ que resultasse em um bom amortecimento das oscilações, isso para todas as condições de operação consideradas.

Dessa forma, objetivando contornar essa dificuldade (de sintonizar $\gamma$ por tentativa e erro), desenvolveu-se neste trabalho uma estratégia adaptativa para ajuste de $\gamma$ em função da condição operacional da planta. $\mathrm{O}$ procedimento pode ser resumido nos seguintes passos:

(i) primeiramente, admite-se que $\gamma$ seja uma função não-linear das condições de operação da planta, isto é $\gamma=\gamma\left(P_{e}, Q_{e}\right)$, e que esta função possa ser aproximada por um sistema fuzzy $\hat{\gamma}\left(P_{e}, Q_{e}\right)$ na

$$
\text { forma } \hat{\gamma}\left(P_{e}, Q_{e}\right)=\frac{\sum_{l=1}^{M} \rho^{(l)}\left(P_{e}, Q_{e}\right) \gamma^{(l)}}{\sum_{l=1}^{M} \rho^{(l)}\left(P_{e}, Q_{e}\right)}
$$

(ii) estima-se então os parâmetros $\gamma^{(l)}, l=1, \ldots, M$, da aproximação fuzzy $\hat{\gamma}\left(P_{e}, Q_{e}\right)$, em (10), através de uma lei de aprendizado obtida da minimização do seguinte critério

$J=\frac{1}{2} \lambda_{1}\left[V_{T R e f}(t+1)-V_{T}(t+1)\right]^{2}+\frac{1}{2} \lambda_{2}[\Delta \omega(t+1)]^{2}$

onde os fatores de ponderação, $\lambda_{1}$ e $\lambda_{2}$, são escalares não-negativos que devem ser selecionados para representar a relação de compromisso existente entre uma rápida recuperação da tensão terminal (manutenção da estabilidade transitória) e o amortecimento das oscilações do rotor (melhoria da estabilidade dinâmica).

\subsubsection{Critérios para Seleção dos Parâmetros da Lei de Aprendizado}

Os parâmetros de aprendizado $\lambda_{1}, \lambda_{2}$, na equaçào (11), e a taxa de aprendizado, $\alpha$ (equações A.12 e A.13), deverão ser previamente selecionados pelo projetista. Para o presente estudo, foram selecionados os valores $\lambda_{1}=0.001$ e $\lambda_{2}=1$, o que significa que atribuiu-se um elevado grau de importância ao amortecimento das oscilações dinâmicas do ângulo do rotor. $\mathrm{O}$ valor da taxa de aprendizado foi selecionado, por tentativa e erro, como sendo $\alpha=0.005$, um valor pequeno para evitar instabilidades no aprendizado.

Finalmente, foi adotado um limite de $\gamma_{\min }=-0.3$ visando não reduzir excessivamente o torque de sincronismo. Em Kundur (1994), é mostrado que um aumento excessivo no torque de amortecimento pode reduzir a magnitude do torque de sincronismo, o que implicaria em uma redução nas margens de estabilidade transitória da planta.

\subsection{Algoritmo para a Execução on-line da Lei de Controle Fuzzy}

Admitindo-se que as etapas do projeto do controlador tenham sido concluídas, o algoritmo que resume a execução on-line do controlador fuzzy é o seguinte (repetido a cada intervalo de amostragem $T_{S}=0.020$ segundos):

(i) Efetuar as medidas de $V_{T}, \Delta \omega, P_{e}$ e $Q_{e}$.

(ii) Calcular os valores das funções de pertinência para os atuais valores das variáveis de operação $P_{e}$ e $Q_{e}$.

(iii) Calcular os presentes valores das funções de validação $\rho^{(l)}\left(P_{e}, Q_{e}\right)$, dos controladores locais, utilizando-se a equação (7).

(iv) Atualizar os valores dos coeficientes da lei de controle fuzzy, para o presente ponto de operação, utilizando-se as equações (8.1), (8.2), e (8.3).

(v) Atualizar o valor do fator estabilizante $\hat{\gamma}\left(P_{e}, Q_{e}\right)$, para o ponto de operação atual, utilizando-se a equação (10).

(vi) Calcular o valor da saída modificada da planta, $y(t)$, utilizando-se a equação (9) com $\gamma=\hat{\gamma}\left(P_{e}, Q_{e}\right)$.

(vii) Atualizar o valor do sinal de controle, $u(t)$, utilizando-se a lei de controle fuzzy (8).

(viii) Aplicar $u(t)$ à planta.

(ix) Aguardar até o próximo intervalo de amostragem, para então repetir os passos de (i) até (ix).

\section{RESULTADOS DE ESTUDOS DE SIMULAÇÃO}

Nesta seção são apresentados alguns dos resultados obtidos nos testes de simulação efetuados para avaliar o desempenho do controlador fuzzy proposto. 


\subsection{Desempenho Obtido na Melhoria da Regulação de Tensão}

Os resultados dos testes para avaliar o desempenho do controlador fuzzy, em relação a uma melhor regulação da tensão terminal, são apresentados na figura 4. A comparação, neste caso, será feita em relação ao desempenho obtido quando se utiliza somente o RAT fixo. A condição de operação inicial para este teste foi: $P_{e}=0.90(\mathrm{pu})$ e $Q_{e}=0.10(\mathrm{pu})$. Em t=1 segundo, aplicouse uma variação do tipo degrau, com amplitude de +0.010 pu, na referência do RAT fixo.
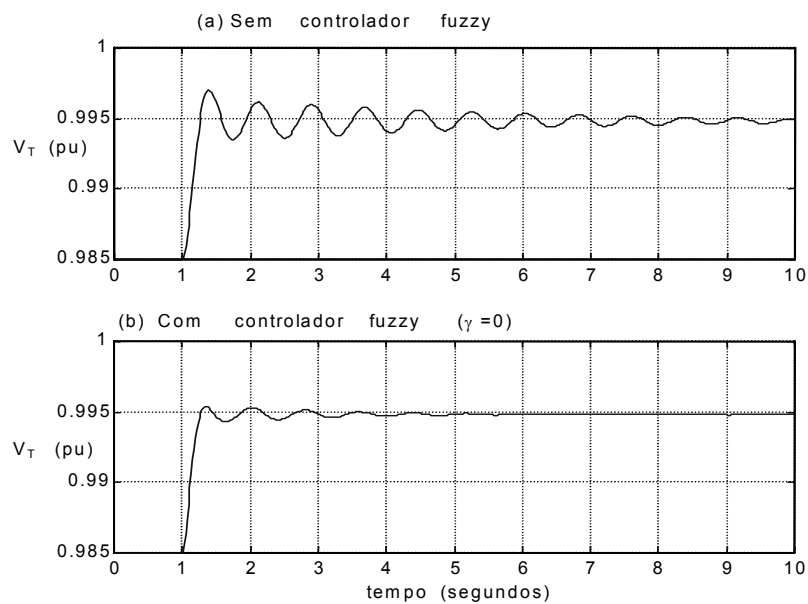

Figura 4.: Respostas para uma variação degrau de 0.010 pu em $V_{\text {Tref. }}$.

Pode ser observado (figura 4.a) que, quando somente o RAT fixo é utilizado, a tensão terminal apresenta um sobressinal de aproximadamente 15 porcento do valor nominal e um tempo de acomodação da ordem de 10 segundos. Este desempenho pobre deve-se ao fato de que o RAT fixo não foi capaz de ajustar seu desempenho de acordo com a nova condição de operação da planta.

A resposta da saída da planta, quando se utiliza o controlador fuzzy é mostrada na figura 4.b, para a mesma variação degrau do caso anterior. Pode ser observado que a regulação da tensão terminal apresenta um desempenho bastante superior, quando se utiliza o controlador fuzzy, do que aquele apresentado quando apenas o RAT fixo é utilizado. A ação suplementar do controlador fuzzy foi capaz, não apenas de reduzir o sobressinal para menos de 5 porcento do valor nominal (como desejado), como também reduziu o tempo de acomodação para menos de 2 segundos, melhorando bastante o desempenho do sistema.

\subsection{Desempenho Obtido no Amortecimento das Oscilações Dinâmicas do Rotor}

Para melhor avaliar o desempenho do controlador fuzzy, quanto à sua capacidade de auxiliar no amortecimento das oscilações dinâmicas do rotor, o seu desempenho foi comparado ao desempenho obtido por um ESP fixo. O ESP fixo, utilizado neste estudo, foi projetado de acordo com as recomendações descritas em Larsen e Swann (1981), de modo a fornecer um amortecimento relativo $\xi=0.30$, sem alterar substancialmente a freqüência angular do modo de oscilação alvo, com a planta operando na condição operacional $\mathrm{P}_{\mathrm{e}}=0.90 \mathrm{pu} \mathrm{e} \mathrm{Q}_{\mathrm{e}}=0.10 \mathrm{pu}$. A simulação foi iniciada com o sistema partindo das mesmas condições operacionais utilizadas para o projeto do ESP fixo $\left(\mathrm{P}_{\mathrm{e}}=0.90 \mathrm{pu}, \mathrm{Q}_{\mathrm{e}}=0.10 \mathrm{pu}, \mathrm{V}_{\mathrm{T} 0}=0.985 \mathrm{pu}, \delta_{O}=61.2^{\circ}\right)$.
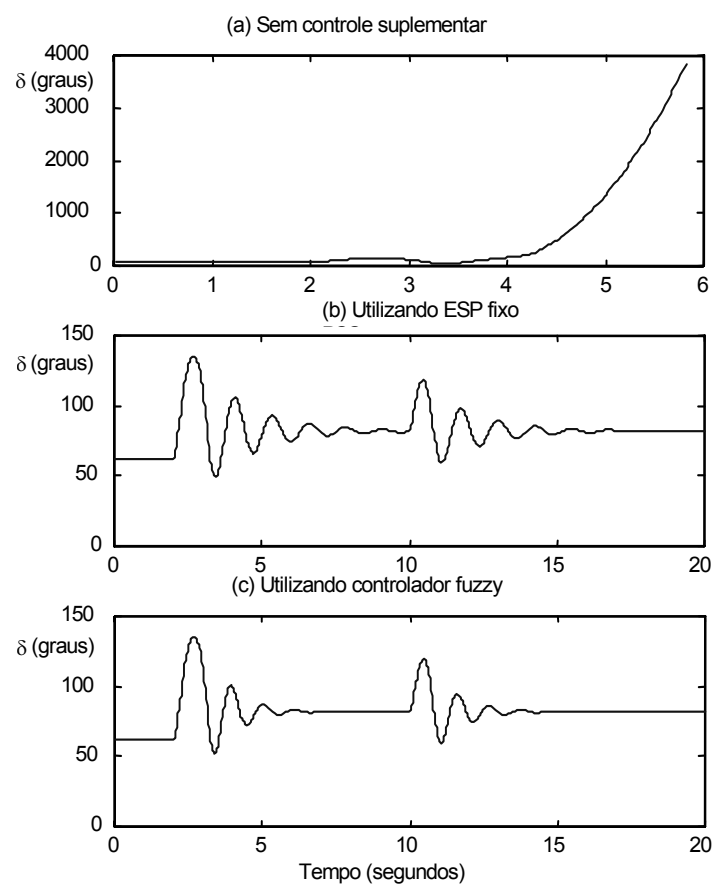

Figura 5. Variação do ângulo de carga (em graus).

Em $\mathrm{t}=2$ segundos (ver figura 5.a), o sistema foi submetido a uma falta do tipo curto-circuito trifásico nos terminais do gerador, com duração de $50 \mathrm{~ms}$. Após o curto-circuito, o sistema perde uma das linhas transmissão. O sistema passa então a operar com apenas com uma das linhas (ou seja, a falta provocou uma substancial variação estrutural na planta). Em $\mathrm{t}=10$ segundos, o sistema é novamente submetido à ocorrência de um segundo curto-circuito trifásico nos terminais da máquina, agora com duração de $50 \mathrm{~ms}$, sendo desta vez não houve perda de linha de transmissão após sanado o curto-circuito. 
Pode ser observado (na figura 5.a) que, sem o emprego de um controle estabilizador, o sistema de potência perderia sua estabilidade imediatamente após a ocorrência da primeira falta. Esta é uma situação típica em que o uso de um controle estabilizador é indispensável para a operação segura do sistema. Pode ser observado, ainda, na figura 5.c, que a ação estabilizadora do controlador fuzzy foi capaz de manter a estabilidade do sistema após a ocorrência de ambas as faltas, com um rápido amortecimento das oscilações dinâmicas do rotor.
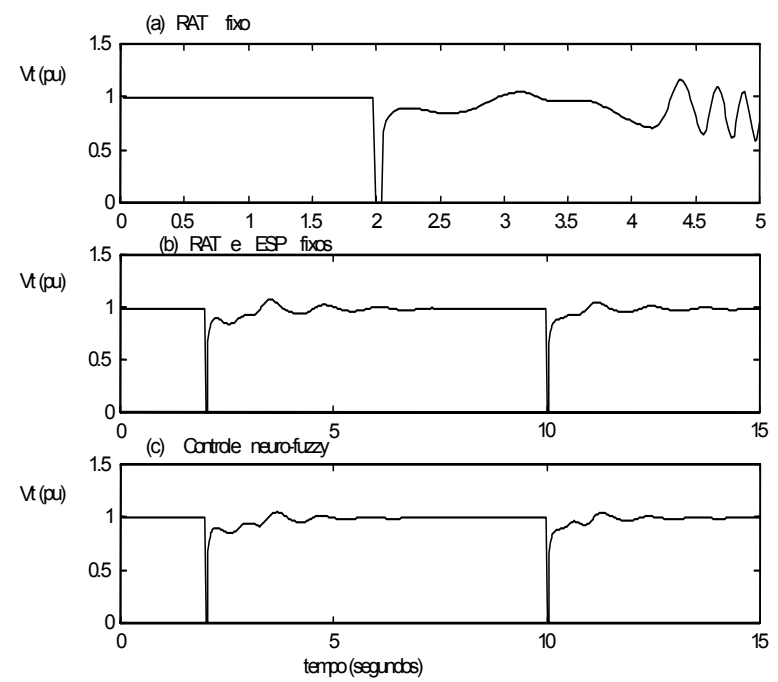

Figura 6. Variação da tensão terminal.

Os resultados mostram que o desempenho do controlador fuzzy foi superior ao desempenho obtido pelo ESP fixo. O ESP fixo, neste caso, sofreu um aconsiderável redução em seu desempenho em razão da variação estrutural sofrida pela planta, com a perda de uma das linhas de transmissão. Note, ainda, que o bom desempenho do controlador fuzzy foi obtido graças à interpolação efetuada entre os ganhos dos seus controladores locais, uma vez que nenhum dos controladores locais foi especificamente projetado para as condições operacionais utilizadas neste experimento. Isto demonstra uma boa capacidade de generalização do controlador fuzzy.

$\mathrm{Na}$ figura 6, pode ser observado que o desempenho superior do controlador fuzzy, em termos do amortecimento das oscilações eletromecânicas, foi conseguido sem o prejuízo de uma rápida recuperação da tensão terminal, após a ocorrência de ambos os curtos circuitos. Ou seja, sem prejudicar a estabilidade transitória da planta.

\section{POSSIBILIDADES E LIMITAÇÕES PARA A APLICAÇÃO DA ESTRATÉGIA EM SISTEMAS MULTIMÁQUINAS}

Os trabalhos de investigação da estratégia de controle fuuzy, proposta neste artigo ficaram, até o presente momento, restritos à avaliações computacionais, com a simulação de sistemas do tipo máquina interligada ao barramento infinito. Os autores pretendem investigar e relatar, em um futuro trabalho, o desempenho da estratégia quando utilizada em um sistema multimáquinas, com vários modos de oscilação local e inter-área. No entanto, baseados na experiência adquirida com o estudo de outras técnicas de controle adaptativo em sistemas multimáquinas (Barreiros et al., 1998), os autores vislumbram as seguintes limitações e potencialidades de aplicação da estratégia em sistemas multimáquinas:

- $\quad$ No caso de um controle descentralizado e nãocoordenado, a estratégia fuzzy poderia ser aplicada, com base apenas na realimentação de sinais mensuráveis localmente. Os controladores fuzzy, neste caso, deveriam ser localizados de modo a ter como alvo um determinado modo de oscilação (local ou interárea), com uma interferência mínima nos demais modos.

Tanto o posicionamento do controlador quanto a seleção de um sinal local (ou de um sinal sintetizado a partir dos sinais locais) mais adequado para realimentação teriam que ser decididos através de um estudo detalhado, baseado em análise modal, procurando obter-se um compromisso razoável entre a seleção de uma localização (gerador) com suficiente controlabilidade para o modo alvo e (preferencialmente) uma baixa controlabilidade para os demais modos de oscilação, de maneira a afeta-los minimamente. Para isso, deveria existir suficiente informação a priori, sobre o sistema multimáquinas, para que o desempenho dos controladores pudesse ser avaliado via simulação, antes de sua efetiva implantação.

Embora as medidas das potências ativa e reativa nos terminais da máquina tenham sido as variáveis de operação utilizadas no caso máquina-barra infinita estudado neste trabalho, ainda não está claro, para os autores, quais seriam as variáveis de operação mais indicadas para uso em um sistema multimáquinas. Uma resposta para este problema certamente será obtida com futuras investigações.

Os avanços recentes na tecnologia de medição sincronizada de fasores (Liu et al. (1999)), nos mecanismos de proteção digital e na crescente velocidade de transmissão de dados via rede Internet abrem um novo leque de possibilidades para que se 
possa testar estratégias avançadas de controle coordenado em sistemas multimáquinas (Fardanesh (2002)).

Os autores reconhecem que ainda existem diversos aspectos que podem ser aprimorados, na estratégia de controle fuzzy apresentada, visando-se um possível teste em um sistema de potência real. Um desses aspectos diz respeito ao problema do aumento exponencial de regras (ou do número de modelos locais) com o número de variáveis de operação sendo utilizadas. Dessa forma, em um sistema de grande porte, uma seleção adequada das variáveis de operação pode não ser uma tarefa trivial. Neste caso, um alternativa seria utilizar-se uma estratégia fuzzy com um sistema de supervisão baseado em modelos locais auto-ajustáveis.

\section{CONCLUSÕES}

Neste trabalho, foi apresentada uma estratégia de controle fuzzy, do tipo rede de controladores locais, destinada a melhorar o desempenho dinâmico de sistemas elétricos de potência. Os ganhos da lei de controle fuzzy são adaptados on-line, de acordo com a atual condição de operação da planta, objetivando-se manter um desempenho uniforme dentro da faixa de condições operacionais permitidas. Foram discutidos os critérios que nortearam a seleção das variáveis de projeto do controlador fuzzy e discutiu-se, ainda, o impacto das variáveis de projeto na estabilidade do sistema em malha fechada.

A seleção das variáveis de projeto foi efetuada levando-se em consideração os compromissos existentes entre a estabilidade em malha fechada, a velocidade de execução, e a capacidade de generalização do controlador fuzzy. Uma lei de aprendizado foi derivada para permitir a estimação do fator de estabilização em função das condições operacionais da planta, o que evitaria a necessidade de ajustes baseados em tentativa e erro.

Os resultados demonstram que o desempenho do sistema, em termos de regulação da tensão e do amortecimento das oscilações dinâmicas, foi melhorado pelo uso da estratégia de controle fuzzy. Uma outra vantagem da estratégia apresentada é que a mesma não necessitaria de um conhecimento a priori detalhado dos valores dos parâmetros físicos do sistema de potência, uma vez que o projeto é baseado no uso de modelos identificados a partir de dados de entrada e saída da planta e, também, em considerações qualitativas a respeito do comportamento dinâmico da planta.

A estratégia apresentada pode ser uma alternativa vantajosa ao uso das tradicionais estratégias de controle adaptativo, do tipo auto-ajustável, uma vez que a capacidade de interpolação provida pela rede fuzzy de controladores locais eliminaria a necessidade do uso de estimadores recursivos operando on-line, aumentando, com isso, a confiabilidade e a robustez do sistema diante da ocorrência de faltas severas e reduzindo sobremaneira a carga de processamento on-line necessária.

Os testes de simulação, executados até o momento, foram efetuados em sistemas do tipo máquina-barra infinita, onde as variáveis utilizadas são acessíveis localmente. Supõe-se que, em princípio, não deveria haver problemas de utilização desta estratégia em sistemas de potência multimáquinas com controle descentralizado, pelo menos no caso não-coordenado, uma vez que, em tais sistemas, os controladores utilizam apenas variáveis medidas localmente.

Um outro aspecto, que demandaria investigação adicional, diz respeito aos possíveis efeitos que poderiam ser provocados pela adaptação do fator $\gamma$ ( equação (9)). Um desses possíveis efeitos seria uma ligeira alteração na posição dos pólos de malha fechada, em relação ao especificado na fase de projeto. Alguns autores têm investigado o problema de projetar controladores de modo a manter a excursão dos pólos de malha fechada sempre restritos à determinadas regiões do plano complexo $\mathrm{Z}$, regiões estas especificadas em termos do desempenho tolerável para o sistema em malha fechada (Lin et al. (1998)). Infelizmente, tal requisito geralmente assume a forma de um problema de otimização, sendo portanto inadequado para cálculos que devam ser efetuados on-line, a cada intervalo de amostragem do controlador (intervalos de amostragem curtos, de algumas dezenas de milisegundos, como no presente caso). Os autores se propõem a investigar mais profundamente este aspecto e relatar os resultados em futuros trabalhos.

No momento, os autores estão concluindo a montagem de um laboratório equipado com um micro-gerador de 10KVA, no qual a estratégia apresentada poderia ser avaliada em um ambiente real, sujeito a perturbações eletromagnéticas e também imperfeições nos sensores e atuadores. Tal implementação constituiria um passo inicial, e essencial, no sentido de recomendar o uso de estratégias de controle adaptativo, baseadas em inteligencia computacional, para futuros testes em geradores nas grandes usinas elétricas.

\section{REFERÊNCIAS BIBLIOGRÁFICAS}

Arrilaga, J. and N. R. Watson (2001). Computer Modelling of Electrical Power Systems. $2^{\text {nd }}$ edition. John Wiley \& Sons, West Sussex, England.

Ästrom, K. J. and B. Wittenmark (1997). Computer Controlled Systems: Theory and Design. $3^{\text {rd }}$ Edition, Prentice-Hall. 
Barra Jr.,W. (2001). Estratégias Neuro-Fuzzy Adaptativas Aplicadas ao Controle de Sistemas Elétricos de Potência. Tese de Doutorado, Programa de pósgraduação em Engenharia Elétrica, Universidade Federal do Pará, Belém, PA.

Barreiros, J. A. L., A. Silva and A. J. A. Simões Costa (1998). A Self-tuning Generalized Predictive Power System Stabilizer. Electrical Power System \& Energy Conversion, Elsevier Science, 20, No.3, pp 213-219.

Costa Jr., C. T. (1999). Méthodes de Commande Adaptative Par Supervision Pour la Régulation d'un Générateur Entraîné par Turbine HydrauliqueThese de Docteur, Institut National Polytechnique de Grenoble, France.

Fardanesh, B. (2002). Futures Trends in Power System Control. IEEE Computer Applications in Power, July, pp. 25-31.

Farsi, M., K. J. Zachariah and J. W. Finch (1997). Implementation of a Self-tunning AVR. IEE Proc. Control Theory Appl. , Special Issue on Adaptive Controllers in Practice, 144, No. 1, January, pp. 32-39.

Flynn, M. S., G. W. Irwin, M. D. Brown, E. Swidenbank and B. W. Hogg (1997). Neural Control of Turbo Generators Systems. Automatica, 33, No. 11, pp. 1961-1973.

Gollee, H. and K. J. Hunt (1997). Nonlinear Modelling and Control of Electrically Stimulated Muscle: A Local Model Network Approach. Int. Journal of Control, 68, No. 6, pp. 1259-1288.

Hunt, K. J. and T. A. Johansen (1997). Design and Analysis of Gain Scheduled Control Using Local Controller Network. Int. Journal of Control, 66, No. 5, pp. 619-651.

Kundur, P. (1994). Power Systems Stability and Control. McGraw-Hill.

Landau, I. D.(19901). System Identification and Control Design Using P.I.M. + Software. Prentice Hall.

Larsen, E. V. and D. A. Swann (1981). Applying Power

Systems Stabilizers. Parts I ,II,III. IEEE Trans. Power Appar. Syst, pp. 3017-3024, pp. 3025-3033, pp. 3034-3046.

Lin, W., W. K. Ho, T. H. Lee, K. V. Ling and W. Xu (1998). Generalized Predictive Controller with Pole
Restriction. IEE Proc. Control Theory Appl., 145, No. 2, March, pp. 219-225.

Liu,C. and S. Tsu(1999). Neuro-fuzzy Approach to RealTime Transient stability Prediction Based on Synchronized Phasor Measurements. Electric Power System Research, 49, pp.123-127.

Prasad, G. , E. Swidenbank and W. Hogg (1998). A Local Model Network Based Multivariable Long-Range Predictive Control Strategy for Thermal Power Plants. Automatica, 33, No. 11, pp.1961-1973.

Zachariah, K. J. (1994). Implementation of Self-tuning Control for Turbine Generators, PH.D. Thesis, Department of Electrical and Electronic Engineering, University of Newcastle-upon-Tyne, U.K.

Zang, J. e A. J. Morris(1995). Fuzzy Neural Networks for Nonlinear Systems Modelling. IEE Proc. Control Theory Appl. , 142, No. 6, November, pp. 551-561.

Wang, L. (1994). Adaptive Fuzzy Systems and ControlDesign and Stability Analysis. Prentice Hall.

Wang, H., G. P. Liu, C. J. Harris and M. Brown(1995). Advanced Adaptive Control. Pergamon Press.

\section{APÊNDICE A - MODELO NÃO-LINEAR DO SISTEMA MÁQUINA - BARRAMENTO INFINITO}

Equações diferenciais:

$$
\begin{gathered}
\frac{d \omega(t)}{d t}=\frac{\omega_{o}}{2 H}\left[P_{m}(t)-P_{e}(t)-D_{o}\left(\omega(t)-\omega_{o}\right)\right] \\
\frac{d \delta(t)}{d t}=\omega(t)-\omega_{o} \\
\frac{d E_{q}^{\prime}(t)}{d t}=\frac{E_{f}+\left(X_{d}-X_{d}^{\prime}\right) I_{d}-E_{q}^{\prime}}{T_{d o}^{\prime}} \\
\frac{d E_{q}^{\prime \prime}(t)}{d t}=\frac{E_{q}^{\prime}+\left(X_{d}^{\prime}-X_{d}^{\prime \prime}\right) I_{d}-E_{q}^{\prime \prime}}{T_{d o}^{\prime \prime}} \\
\frac{d E_{d}^{\prime \prime}(t)}{d t}=\frac{-\left(X_{q}-X_{q}^{\prime \prime}\right) I_{q}-E_{d}^{\prime \prime}}{T_{q o}^{\prime \prime}}
\end{gathered}
$$

Equações algébricas:

$$
\begin{gathered}
E_{q}^{\prime \prime}=V_{q}+R_{a} I_{q}-X_{d}^{\prime \prime} I_{d} \\
E_{d}^{\prime \prime}=V_{d}+R_{a} I_{d}+X_{q}^{\prime \prime} I_{q} \\
P_{e}=V_{d} I_{d}+V_{q} I_{q}+R_{a}\left(I_{d}^{2}+I_{q}^{2}\right)
\end{gathered}
$$




$$
\begin{gathered}
Q_{e}=V_{q} I_{d}-V_{d} I_{q} \\
V_{d}=X_{e} I_{q}+R_{e} I_{d}-V_{\infty} \operatorname{sen} \delta \\
V_{q}=R_{e} I_{q}-X_{e} I_{d}+V_{\infty} \cos \delta
\end{gathered}
$$

onde:

$\omega, \omega_{\mathrm{o}}$ : velocidades angulares instantânea e síncrona $(\mathrm{rad} / \mathrm{seg})$;

$\omega \mathrm{H}, \mathrm{D}_{0}$ : constante de inércia (seg) e de amortecimento da máquina (pu de potência./rad/seg);

$\omega P_{m}, P_{e}, Q_{e}$ : potências mecânica, elétrica ativa e elétrica reativa $(\mathrm{pu})$;

$\delta$ : ângulo do rotor (rad);

$E_{q}^{\prime}, E_{q}^{\prime \prime}, E_{d}^{\prime \prime}$ : tensões transitórias e subtransitórias dos eixos d e q (pu);

$V_{q}, I_{q}, V_{d}$ e $I_{d}$ : tensões e correntes nos eixos q e d $(\mathrm{pu}) ; x_{d}, x_{d}^{\prime}, x_{d}^{\prime \prime}, x_{q}, x_{q}^{\prime \prime}$ : reatâncias síncronas, transitórias, e subtransitórias dos eixo d e q (pu);

$T_{d o}^{\prime}$ e $T_{d o}^{\prime \prime}$ : constantes de tempo transitória e subtransitória de circuito aberto do eixo d (seg);

$E_{f}$ : tensão de campo aplicada $(\mathrm{pu})$;

$R_{a}$ : resistência de armadura $(\mathrm{pu})$;

$R_{e}, X_{e}$ : resistência e reatância equivalentes da linha de transmissão $(\mathrm{pu})$;

$V_{\infty}$ é a magnitude da tensão no barramento infinito (pu).

\section{APÊNDICE B - VALORES DOS PARÂMETROS UTILIZADOS NA SIMULAÇÃO}

Parâmetros do Gerador Síncrono e do sistema de excitação: $X_{d}=1.445 \mathrm{pu} ; X_{q}=0.959 \mathrm{pu} ; X_{d}^{\prime}=0.316$ $\mathrm{pu} ; \quad X_{d}^{\prime \prime}=0.179 \mathrm{pu} ; \quad X_{q}^{\prime \prime}=0.162 \mathrm{pu} ; \quad R_{a}=0.001 \mathrm{pu}$; $T_{d 0}^{\prime}=5.256$ Seg.; $T_{d 0}^{\prime \prime}=0.0282$ Seg.; $T_{q 0}^{\prime \prime}=0.157$ Seg.; $H=4.27 \quad$ Seg.; $D_{0}=0 ; \quad K_{E}=10 ; \quad T_{E}=0.010 \mathrm{seg}$; $V_{E M A X}=6 \mathrm{pu} ; V_{E M I N}=-6 \mathrm{pu}$

Parâmetros equivalentes da linha de transmissão dupla :
$R_{e}=0.02 \mathrm{pu} ; X_{e}=0.415 \mathrm{pu}$

Parâmetros do Regulador PID digital fixo:

$r_{0}=57.793 ; r_{1}=-88.207 ; r_{2}=-33.72 ; s_{1}=0.6833$

\section{APÊNCICE C- DERIVAÇÃO DA LEI DE APRENDIZADO PARA OS PARÂMETROS $\gamma^{(I)}$}

É mostrado em (Wang,1994) que uma lei de aprendizado do tipo $\gamma_{k+1}^{(l)}=\gamma_{k}^{(l)}-\alpha \partial J / \partial \gamma^{(l)}$, com $0<\alpha<1$, pode minimizar uma função custo na forma dada pela equação (11).

Dessa forma, expandindo-se a derivada parcial $\partial J / \partial \gamma^{(l)}$, através do uso da regra da derivação em cadeia, e utilizando-se os coeficientes $b_{O V}^{(l)}$ e $b_{O \omega}^{(l)}$ ( os coeficientes $b_{0}$ dos modelos locais identificados para os canais de tensão terminal e de velocidade angular, respectivamente) como aproximações para as derivadas parciais $\partial V_{T} / \partial u$ e $\partial \Delta \omega / \partial u$, respectivamente, e, ainda, utilizando-se os modelos locais dos canais de tensão terminal e de velocidade angular como preditores para $V_{T}(t+1) \mathrm{e}$ $\Delta \omega(t+1)$, na função custo (11), obtém-se, então, a seguinte lei de aprendizado para sintonia automática dos parâmetros $\gamma^{(l)}$ (Barra Jr, 2001)

$$
\gamma^{(l)}(t)=\gamma^{(l)}(t-1)+\alpha x^{(l)}(t), \quad \gamma_{\min }<\gamma^{(l)}(t) \leq 0
$$

com

$$
x^{(l)}(t)=\left(\lambda_{2} b_{0 \omega}^{(l)} \Delta \omega(t)-\lambda_{1} b_{0 V}^{(l)}\left(V_{T r e f}(t)-V_{T}(t)\right)\right) R^{(l)}\left(q^{-1}\right) \Delta \omega(t-1)
$$

onde o escalar $\gamma_{\min }\left(-1<\gamma_{\min }<0\right)$ especifica um limite inferior para os parâmetros $\gamma^{(l)}$ durante o aprendizado, $\alpha(0 \leq \alpha<1)$ é a taxa de aprendizado, e $R^{(l)}\left(q^{-1}\right)$ é o polinômio de realimentação do $l$-ésimo controlador local

(equações (3) e (3.1)). Os parâmetros $\gamma^{(l)}$ devem ser calculados "off-line", utilizando-se para isso as equações (A .12) e (A.13), e utilizando-se os modelos locais como preditores das saídas da planta.

\section{APÊNDICE D- NÚMERO DE REGRAS COM ATIVAÇÃO NÃO-NULA}

Conforme mostrado na equação (7), em um sistema fuzzy, quando se utiliza inferência do tipo produto, o peso de cada regra é dado pelo produto dos valores não-nulos 
assumidos pelas funções de pertinência calculadas no ponto de operação considerado. Para exemplificar seja um espaço de operação composto por duas variáveis de operação $x, y$ onde os universos de discurso estejam particionados em $n_{x}$ e $m_{y}$ conjuntos fuzzy $\left[X_{1}, X_{2}\right.$,

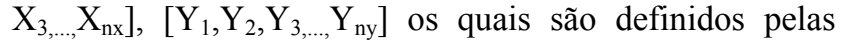
respectivas funções de pertinência $\left[\mu_{\mathrm{X} 1}(\mathrm{x}), \mu_{\mathrm{X} 2}(\mathrm{x})\right.$, $\left.\mu_{\mathrm{X} 3}(\mathrm{x}), \ldots, \mu_{\mathrm{Xnx}}(\mathrm{x})\right],\left[\mu_{\mathrm{Y} 1}(\mathrm{y}), \mu_{\mathrm{Y} 2}(\mathrm{y}), \mu_{\mathrm{Y} 3}(\mathrm{y}), \ldots, \mu_{\mathrm{Yny}}(\mathrm{y})\right] . \mathrm{O}$ número total de regras corresponde ao número de elementos do produto cartesiano $\left[\mathrm{X}_{1}, \mathrm{X}_{2}, \mathrm{X}_{3, \ldots}\right.$, $\left.\mathrm{X}_{\mathrm{nx}}\right] \mathrm{x}\left[\mathrm{Y}_{1}, \mathrm{Y}_{2}, \mathrm{Y}_{3, \ldots}, \mathrm{Y}_{\mathrm{ny}}\right]$, o que resulta em total $\mathrm{N}_{\text {regras }}=$ $n_{X} . n_{Y}$ regras, cujos valores $\mathrm{e}$ ativação são na forma $\mu_{X i}(x) . \mu_{Y j}(x)$, com $\mathrm{i}=1,2,3, . ., \mathrm{n}_{\mathrm{x}} \mathrm{e} \mathrm{j}=1,2,3, \ldots, \mathrm{n}_{\mathrm{y}}$. Suponhase que as funções de pertinência sejam selecionadas de tal modo que, para um dado ponto de operação $(x, y)$, apenas duas funções de pertinência por variável de operação tenham valor não nulo no ponto $(x, y)$, as funções de pertinência $\mu_{X_{i}}(x)$ e $\mu_{X_{i+1}}(x)$, para a variável $x$, e as funções de pertinência $\mu_{Y_{j}}(y)$ e $\mu_{Y_{j+1}}(y)$, para a variável y. Dessa forma, de um total de $n_{X} . n_{Y}$ valores de ativação calculados no ponto $(x, y)$, teremos somente os seguintes valores de ativação não-nulos $\left[\mu_{X_{i}}(x) \cdot \mu_{Y_{j}}(y), \mu_{X_{i}}(x) \cdot \mu_{Y_{j+1}}(y), \mu_{X_{i+1}}(x) \cdot \mu_{Y_{j}}(y), \mu_{X_{i+1}}(x)\right.$.

$\left.\mu_{y_{j+1}}(y)\right]$, ou seja, apenas um subconjunto com $2^{2}$ regras (do total de $n_{X} . n_{Y}$ ) possuirá valores de ativação não-nulos. Este resultado pode ser facilmente generalizado para casos com $\mathrm{N}$ variáveis de operação, resultando um subconjunto $\operatorname{com} 2^{\mathrm{N}}$ regras com valores de ativação não-nulos.

\section{APÊNDICE E - FORMA CANÔNICA RST PARA REPRESENTAÇÃO DE CONTROLADORES DIGITAIS}

A forma polinomial da lei de controle, expressa pelas equações (3), (3.1),(3.1) e (3.3), é conhecida como forma canônica RST e é uma representação genérica para controladores digitais (Landau, 1990, Capítulo 2). Diversos tipos de controladores digitais, incluindo PID digitais, podem ser representados na forma RST. O polinômio $\mathrm{R}$ é o polinômio que representa a realimentação da saída da planta, y, e de seus valores passados. O polinômio $\mathrm{S}$ representa a realimentação dos valores passados do sinal de saída do controlador, u. O polinômio $\mathrm{T}$ é um polinômio que pondera o valor presente e passados do sinal de referência do controlador. Se o objetivo do controle for obter regulação da variável de saída y, então geralmente escolhe-se $\mathrm{T}=\mathrm{R}$. Quando é desejado um ganho unitário em regime permanente, o polinômio $\mathrm{T}$ deve ser selecionado de acordo com a equação (3.3). 